\title{
Non Local Means Denoising for 3D MR Images
}

\author{
Hao Song \\ School of Electronic Engineering \\ University of Electronic Science and Technology of China \\ Chengdu, China \\ a6802739@163.com
}

\begin{abstract}
Denoising is a crucial step to increase image conspicuity and to improve the performances of all the processing needed for quantitative image analysis. In this paper, the main method we proposed is Non Local Means. We carried out our experiments based on 3D MR images from Brain Web. We compared The NL-means with some classical methods, such as Anisotropic Diffusion Filter and Bilateral Filter. The results show that the Filtering performance of NLmeans is better than other methods. Moreover, we present an optimized version of original NL-means and parallelize the computation on GPU device.
\end{abstract}

Keywords-NL-means; denoising; 3D; MRI; parallelize; GPU

\section{INTRODUCTION}

The goal of image denoising methods is to recover the original image from a noisy measurement,

$$
v(i)=u(i)+n(i)
$$

Where $v(i)$ is the observed value, $u(i)$ is the "true" value and $n(i)$ is the noise perturbation at a voxel $i$.

Therefore, one critical issue concerns the problem of noise removal while keeping the integrity of relevant image information. This is particularly true for various MRI sequences especially when they are acquired on new high field 3T systems. With such devices, along with the improvement of tissue contrast, 3T MR scans may introduce additive artifacts (noise, bias field, geometrical deformation). This increase of noise impacts negatively on quantitative studies involving segmentation and/or registration procedures. This paper focuses on one critical aspect, image denoising, by introducing a new restoration scheme in the 3D medical imaging context. The proposed approach is based on the method originally introduced by Buades [1] but with specific adaptations to medical images. To limit a highly expensive computational cost due to the size of the 3D medical data, we propose an optimized and parallelized implementation.

The paper is structured as follows: Section 2 presents a main overview of the Non Local (NL) means algorithm and a optimized version of the original method, Section 3 presents the experiments result, histogram comparison, visual assessment, and NL-means for 3T MR image. Section 4 is our conclusion for this paper.
Jing Jin

School of Communication and Information Engineering University of Electronic Science and Technology of China Chengdu, China elise jing@163.com

\section{THE NON LOCAL MEANS ALGORITHM}

First, we give a short description of NL-means. Supposed that we are going to deal with a 3D MR image $(256 \times 256 \times 256)$, we can divide NL-means into three main steps:

Step1. For each voxel $i$ in the MR image, we should allocate a search volume $V_{i}(11 \times 11 \times 11)$. For each voxel $j$ in search volume $V_{i}$, we compare the similarity between the local neighborhoods $v\left(N_{j}\right)$ of voxel $j$ with the local neighborhoods $v\left(N_{i}\right)$ of center voxel $i$.

Step2. If the similarity is higher, we can obtain a higher weight $w(i, j)$.

Step3. The denoised center voxel value $v(i)$ is represented by the summation of $v(j) \times w(i, j)$ for each voxel $j$ in the search volume $V_{i}$.

Then, we'll discuss NL-means in detail and present the improvement of the original methods.

\section{A. The original NL-means}

First introduced by Buades in [1], the Non Local means algorithm allows us to avoid the well-known artifacts of the commonly used neighborhood filters. Given a discrete noisy image $v=\{v(i) \mid i \in I\}$, the restored intensity of the voxel $i, N L(v)(i)$ is computed as a weighted average of all the pixels in the image,

$$
N L(v)(i)=\sum_{j \in I} w(i, j) v(j)
$$

Where the family of weights $\{w(i, j)\}_{j}$ depends on the similarity between the voxel $i$ and $j$, and satisfy the usual conditions $w(i, j) \in[0,1]$ and $\sum_{j \in I} w(i, j)=1$.

The number of voxels taken into account in the weighted average can be restricted in a neighborhood that is called in the following "search volume" $V_{i}$ of size $(2 M+1)^{3}$, centered at the current voxel $i$. In this search volume $V_{i}$, the similarity between $i$ and $j$ depends on the similarity of their local neighborhoods $N_{i}$ and $N_{j}$ of size $(2 d+1)^{3}$. 


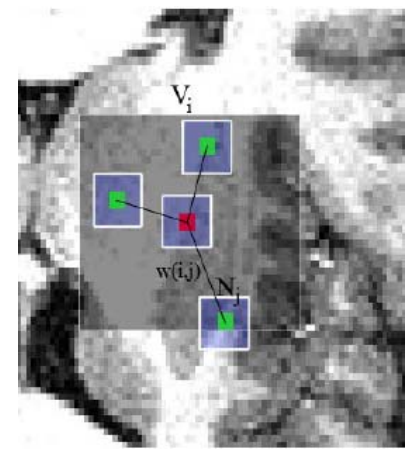

Figure 1. 2D illustration of the NL-means principle . The restored value of voxel $i$ (in red) is a weighted average of all intensities of voxels $j$ in the

search volume $V_{i}$, according to the similarity of their intensities neighborhood $v\left(N_{i}\right)$ and $v\left(N_{j}\right)$

The similarity is measured as a decreasing function of the weighted Euclidean distance $\left\|v\left(N_{i}\right)-v\left(N_{j}\right)\right\|_{2, a}^{2}$, where $a>0$ is the standard deviation of the Gaussian kernel. The application of the Euclidean distance to the noisy neighborhoods raises the following equality,

$$
E\left\|v\left(N_{i}\right)-v\left(N_{j}\right)\right\|_{2, a}^{2}=\left\|u\left(N_{i}-u\left(N_{j}\right)\right)\right\|_{2, a}^{2}+2 \delta^{2}
$$

This equality shows the robustness of the algorithm since in expectation the Euclidean distance conserves the order of the similarity between voxels.

These distances are weighted by the function defined as follows:

$$
w(i, j)=\frac{1}{Z(i)} e^{-\frac{\left\|v\left(N_{i}\right)-v\left(N_{j}\right)\right\|_{2, a}^{2}}{h^{2}}}
$$

Where $Z(i)$ is the normalization coefficient,

$$
Z(i)=\sum_{j} e^{-\frac{\left\|v\left(N_{i}\right)-v\left(N_{j}\right)\right\| \frac{2}{2}, a}{h^{2}}}
$$

From formulation (4), we can clearly see that $0 \leq w(i, j) \leq 1$ and $\sum_{j \in I} w(i, j)=1$.

\section{B. The improvement of the original algorithm}

The optimized version of the Non Local Means is proposed by Pierrick [2]. The complexity of the original algorithm is very huge, especially on 3D data. Let us denote by $N^{3}$ the size of the 3D image, and then the complexity of the algorithm is in the order of $O\left((N(2 M+1)(2 N+1))^{3}\right)$. For a classical MR image data $181 \times 217 \times 181$ voxels, with the smallest value of $d=1$, and $M=5$, the computational time reaches up to 6 hours. This time is far beyond a reasonable duration expected for a denoising algorithm in a medical practice, and thus the reduction of complexity is crucial in the medical context. To reduce the number of voxels taken into account in the weighted average, we can select only the voxels $j$ in $V_{i}$ that will have highest weights $w(i, j)$ in (4) without having to compute the Euclidean distance between $v\left(N_{i}\right)$ and $v\left(N_{j}\right)$. In [5], Mahmooudi propose a method to preselect a s set of the most pertinent voxels $j$ in volume $V_{i}$. This selection is based on the similarity of the mean and the gradient of $v\left(N_{i}\right)$ and $v\left(N_{j}\right)$ : intuitively, similar neighborhoods tend to have close means and close gradients. Since the gradient is sensitive to noise level. In [2], Pierrick proposes that the standard deviation is preferable in case of high level of noise. In that way, the maps of local means and local standard deviations are precomputed in order to avoid repetitive calculations of moments for one same neighborhood. The selection tests can be expressed as follows: if $u_{1}<\frac{\overline{v\left(N_{i}\right)}}{\overline{v\left(N_{j}\right)}}<u_{2}$ and $\delta_{1}^{2}<\frac{\operatorname{var}\left(v\left(N_{i}\right)\right)}{\operatorname{var}\left(v\left(N_{j}\right)\right)}<\delta_{2}^{2}$, $w(i, j)=\frac{1}{Z(i)} e^{-\frac{\left\|v\left(N_{i}\right)-v\left(N_{j}\right)\right\|_{2, a}^{2}}{h^{2}}}$. In other case, $w(i, j)=0$.

\section{Parallelized computation based on GPU Acceleration}

The intrinsic nature of the NL-means algorithm allows to use multithreading, and thus to parallelize the operations. The threads we allocate in the GPU kernel is the slice size of the 3D MR image. We divide the volume into slices, each of them being treated separately by one kernel. We carry out the parallelization computation on the device GeForce GTS 250 .

\section{RESULTS}

In order to evaluate the performance of the NL-means algorithm on 3D T1 MR Images, tests are performed on the Brain Web database composed of $181 \times 217 \times 181$ image. The evaluation framework is based on comparisons with other denoising methods: Anisotropic Diffusion Filter [7] and Bilateral Filter [8]. In all experiments, the typical values used for the NL-means parameters are $d=1, M=5, u_{1}=0.95, u_{2}=1.05, \delta_{1}^{2}=0.5, \delta_{2}^{2}=1.5$, and $h$ is close to the standard deviation of the added noise, influencing the smoothness of the global solution. To obtain a significant improvement in the results, $d$ can be increased, but it implies to increase $M$ yielding to a prohibitive computational time.

\section{A. Histogram comparison}

We compared the histograms of the denoised images with different denoising methods. It is shown that the NL- 
means is the only method able to retrieve a similar histogram as the original image.

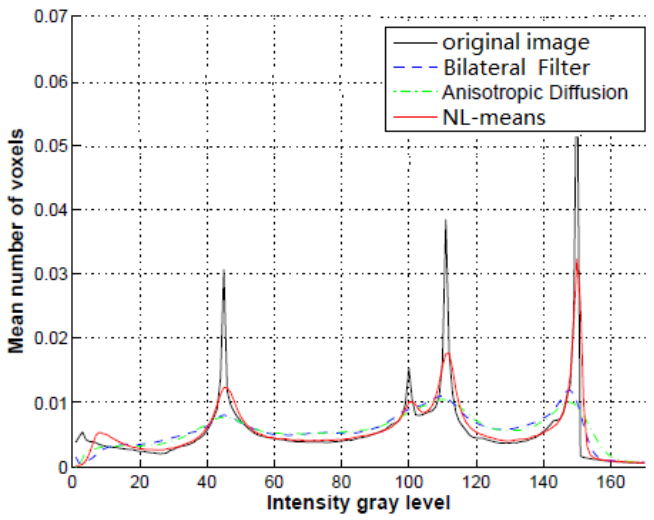

Figure 2. Histograms of the restored images and of the original image. The histogram of the NL-means restored image better fits to the original image

\section{VISUAL ASSESSMENT}

Fig. 3 shows the restored images and the removed noise obtained with three compared methods. As shown in the previous analysis, we can observe that NL-means has the best denoising performance. Moreover, if we focus on the nature of the removed noise, it clearly appears that NL-means restoration preserves better the high frequencies components of the image (i.e. edges).
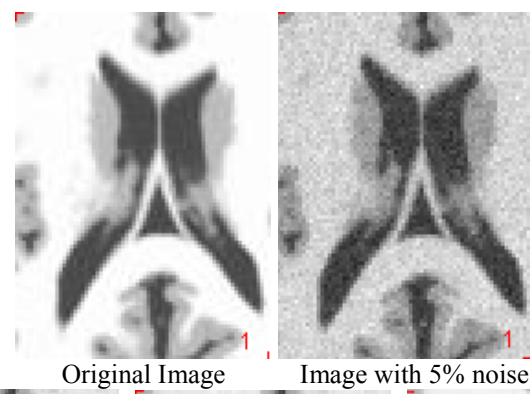

Image with $5 \%$ noise

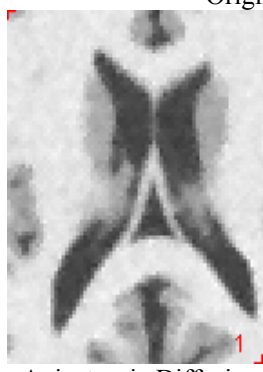

Anisotropic Diffusion

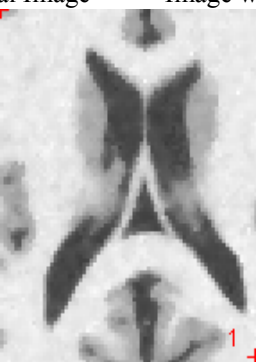

Bilateral Filter

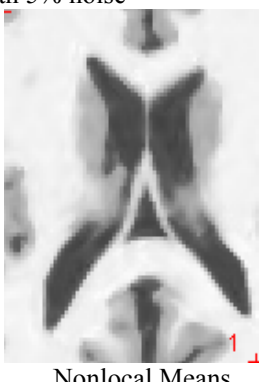

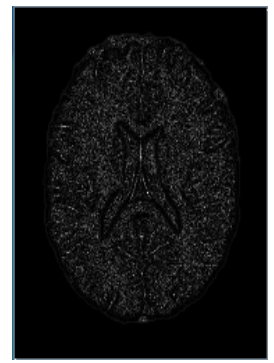
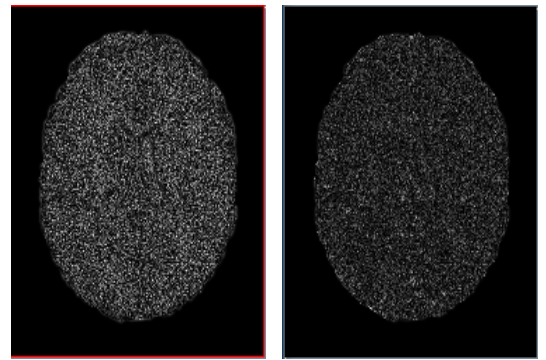

Figure 3. Top: details of the original image and noisy image with a noise level of $9 \%$. Middle: details of the denoised images obtained via three compared methods. Bottom: Images between noisy images and denoised images.

\section{A. NL-means for clinical data}

To show the efficiency of the NL-means algorithm on real data, tests have been performed on a high field MR system (3T). In these images, the gain in resolution being obtained at the expense of an increase of the level of noise, the denoising step is particularly important. The restoration results, presented in Fig.4 show good preservation of the basal ganglia.
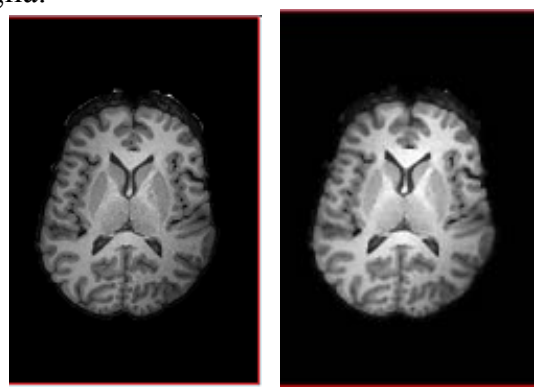

Original 3T image

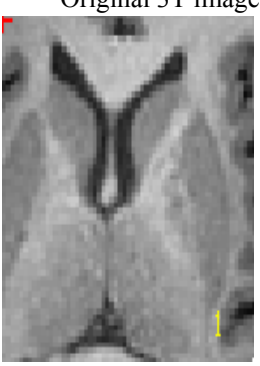

Restored image

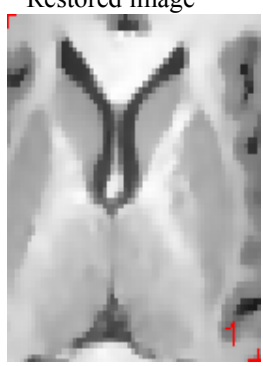

Figure 4. NL-means restoration of 3T MRI data. From left to right: Original image, denoised image.

\section{CONCLUSION AND FURTHER WORK}

This paper applies Non Local means to 3D medical data, and presents an optimized version of the original NL-means. The validations performed on Brain Web dataset bring to the fore how the NL-means denoising outperforms well established other methods, such as Anisotropic Diffusion and Bilateral Filter. We can clearly see that this method is excellent. Its computation complexity is still a challenging problem. The proposed optimized implementation, with voxel pre-selection and multithreading, considerably 
decreases the required computational time. The impact of the NL-means denosing on the performances of postprocessing algorithm, like segmentation and registration schemes need also to be further investigated.

\section{REFERENCES}

[1] A. Buades, B. Coll,and J. M. Morel, "A review of image denoising algorithms, with a new one." Multiscale Modeling \& Simulation, 4(2):490-530, 2005.

[2] Pierrick, Pierre, and Christian, "Fast Non Local Means Denoising for 3D MR Images" In Int. Conf. on Medical Image Computing and Computer Assisted Intervention (MICCAI'06), IRISA campus Beaulieu 35042 Rennes Cedex, France.

[3] J. Boulanger, Ch. Kervrann, and P. Bouthemy. "Adaptive spatiotemporal restoration for $4 \mathrm{~d}$ fluoresence microscopic imaging." MICCAI'05, Palm Springs, USA.
[4] I. S. Jacobs and C. P. Bean, "Fine particles, thin films and exchange anisotropy," in Magnetism, vol. III, G. T. Rado and H. Suhl, Eds. New York: Academic, 1963, pp. 271-350.

[5] M. Mahmoudi and G. Sapiro, "Fast image and video denoising via non-local means of similar neighborhoods" IMA Preprint Series, 2052, 2005.

[6] P. Perona and J. Malik, "Scale-space and edge detection using anisotropic diffusion" IEEE Trans. Pattern Anal. Mach. Intell., 12(7):629-639, 1990.

[7] P. Perona and J. Malik, "Scale-space and edge detection using anisotropic diffusion" IEEE Trans. Pattern Anal. Mach. Intell., 12(7):629-639, 1990.

[8] C. Tomasi and R. Manduchi, "Bilateral Filtering for Gray and Color Images" Proceedings of the 1998 IEEE International Conference on Computer Vision, Bombay, India 